\title{
K-Ras and MSI: Potential Markers of Both Patient Prognosis and Treatment Efficacy
}

\author{
Kiran Turaga, MD and David Shibata, MD \\ Department of Gastrointestinal Oncology, Moffitt Cancer Center, Tampa, FL
}

In this era of growing molecular sophistication and interest in genome-wide analyses to develop prognostic signatures, it is ironic that some of the most promising recent developments in predictive markers for colorectal cancer stem from the re-emergence of interest in two wellestablished and characterized molecular alterations. In this issue of Annals of Surgical Oncology, Nash et al. present two very interesting studies; the first examines the association of microsatellite instability (MSI) and K-ras mutation status with prognosis in patients with colorectal cancer, ${ }^{1}$ while the second evaluates the impact of K-ras (and Ki-67) on the rate and extent of progression of hepatic metastases from colorectal cancer. ${ }^{2}$ Although done in retrospective fashion, both studies report on a substantial number of patients ( $n=532$ and 188, respectively) and the authors add further credence to previously reported associations for these particular genetic alterations.

MSI, found in approximately $20 \%$ of patients with colorectal cancer, has been linked with favorable outcome in young patients. ${ }^{3}$ Using a modified three-locus assay for MSI (BAT25, BAT26, and D2S123), Nash et al. confirm this association, and demonstrate a significant survival advantage (5-year survival 92\% versus 59\%, $P<0.001$ ), which is more apparent in stage II patients. As described previously, patients with MSI tend to have more poorly differentiated, mucin-producing tumors, which are more often found proximal to the splenic flexure, characteristics which were echoed in this study. This survival benefit was borne out in a multivariate hazards model even after adjusting for stage. Although the retrospective nature of this study and the heterogeneity of therapy may lead to bias, this result is not surprising, given the strong association of MSI

(C) Society of Surgical Oncology 2009

Published Online: 20 November 2009

D. Shibata, MD

e-mail: david.shibata@moffitt.org with patient outcome. Of substantial current interest is that, in addition to being a prognostic marker, several highprofile studies have described MSI as a predictive marker of treatment that may predict variable response to 5-flourouracil (5-FU)-based chemotherapy. ${ }^{4-6}$ This association is being further explored as a part of the Eastern Cooperative Oncology Group (ECOG) 5202 trial that is selecting stage II colorectal cancer patients for adjuvant therapy based on MSI and $18 \mathrm{q}$ loss-of-heterozygosity ( $\mathrm{LOH}$ ) status. The current study by Nash et al. is not able to address this specific issue; however, they do contribute to a strong argument that MSI may be critical for both patient prognosis and prediction of treatment outcome.

Similarly, K-ras has received substantial recent attention given multiple reports of mutated K-ras being critical as a predictive factor for lack of response to epidermal growth factor receptor inhibitors such as cetuximab and panitumumab in patients with colorectal cancer. ${ }^{7-10}$ Moreover, K-ras mutations on codon 12 have been associated with unfavorable outcomes, especially in those individuals with advanced disease. K-ras remains one of the commonly mutated oncogenes in human cancers, and the association of K-ras with colorectal cancer prognosis, independent of stage, is controversial. ${ }^{11,12}$ Nash et al. demonstrate in their study that K-ras is independently associated with worse outcome (5-year survival $55 \%$ versus $68 \%$ ), with effects being more pronounced in stage II and III disease. An interesting and novel aspect of the papers, although slightly weakened by the lack of an a priori hypothesis, is the combined analysis of MSI and K-ras in determining the prognosis of colorectal cancer patients, which has not been previously described. In addition, the authors demonstrate an association of K-ras mutation with worse outcomes and accelerated progression of colorectal liver metastasis in patients having undergone surgical resection. The retrospective nature of these analyses is also associated with significant heterogeneity of treatment, which may lend bias 
to their conclusions. Nonetheless, the authors should be commended for their meticulous analysis of a large number of patients and samples and their results are certainly quite compelling. Once again, it is very interesting that both $\mathrm{K}$-ras and MSI may serve roles in predicting survival as well as in treatment selection.

In this era of targeted therapies and personalized medicine, it is clear that molecular markers will play a substantial role in determining both patient treatment and outcomes. However, the field of molecular prognostication can be fraught with the risk of inaccuracy, and promising results must be rigorously validated in prospective studies before incorporating the use of such markers into standard practice.

\section{REFERENCES}

1. Nash GM, Gimbel M, Shia J, et al. KRAS mutation correlates with accelerated metastatic progression in patients with colorectal liver metastases. Ann Surg Oncol. 2009. doi:10.1245/s10434-0090605-3.

2. Nash GM, Gimbel M, Cohen AM, et al. KRAS mutation and microsatellite instability: two genetic markers of early tumor development that influence the prognosis of colorectal cancer. Ann Surg Oncol. 2009. doi:10.1245/s10434-009-0713-0.

3. Gryfe R, Kim H, Hsieh ET, et al. Tumor microsatellite instability and clinical outcome in young patients with colorectal cancer. N Engl J Med. 2000;342(2):69-77.

4. Ribic CM, Sargent DJ, Moore MJ, et al. Tumor microsatelliteinstability status as a predictor of benefit from fluorouracil-based adjuvant chemotherapy for colon cancer. $N$ Engl J Med. 2003; 349(3):247-57.

5. Des Guetz G, Schischmanoff O, Nicolas P, Perret GY, Morere JF, Uzzan B. Does microsatellite instability predict the efficacy of adjuvant chemotherapy in colorectal cancer? A systematic review with meta-analysis. Eur J Cancer. 2009;45(10):1890-6.

6. Bertagnolli MM, Niedzwiecki D, Compton CC, et al. Microsatellite instability predicts improved response to adjuvant therapy with irinotecan, fluorouracil, and leucovorin in stage III colon cancer: Cancer and Leukemia Group B Protocol 89803. J Clin Oncol. 2009;27(11):1814-21.

7. Peeters M, Siena S, Van Cutsem E, et al. Association of progression-free survival, overall survival, and patient-reported outcomes by skin toxicity and KRAS status in patients receiving panitumumab monotherapy. Cancer. 2009;115(7):1544-54.

8. Van Cutsem E, Kohne CH, Hitre E, et al. Cetuximab and chemotherapy as initial treatment for metastatic colorectal cancer. $N$ Engl J Med. 2009;360(14):1408-17.

9. Lievre A, Bachet JB, Boige V, et al. KRAS mutations as an independent prognostic factor in patients with advanced colorectal cancer treated with cetuximab. J Clin Oncol. 2008;26(3): 374-9.

10. Amado RG, Wolf $M$, Peeters $M$, et al. Wild-type KRAS is required for panitumumab efficacy in patients with metastatic colorectal cancer. J Clin Oncol. 2008;26(10):1626-34.

11. Ahnen DJ, Feigl P, Quan G, et al. Ki-ras mutation and p53 overexpression predict the clinical behavior of colorectal cancer: a Southwest Oncology Group study. Cancer Res. 1998;58(6): 1149-58.

12. Samowitz WS, Curtin K, Schaffer D, Robertson M, Leppert M, Slattery ML. Relationship of Ki-ras mutations in colon cancers to tumor location, stage, and survival: a population-based study. Cancer Epidemiol Biomarkers Prev. 2000;9(11):1193-7. 